\title{
High-Contrast Visualization of Endogenous Proteins for Live Imaging
}

Dale A. Fortin ${ }^{1}$, Joshua B. Melander ${ }^{1,3}$, Bart C. Jongbloets ${ }^{1}$, Wei-Hong, Xiong ${ }^{1}$, Caiying Guo ${ }^{2}$, Tianyi Mao $^{1}$ and Haining Zhong ${ }^{1 *}$

1. Vollum Institute, Oregon Health \& Science University, Portland, OR, USA.

2. Howard Hughes Medical Institute, Janelia Research Campus, Ashburn, VA, USA.

3. Present address: Stanford Neurosciences Program, Stanford University, Stanford, CA, USA.

* Corresponding author: zhong@ohsu.edu

Fluorescence imaging of most cellular structures and protein species relies on the proper and specific introduction of fluorescent labels. However, the prevalent protein labeling methods have significant limitations. Immunolabeling does not usually allow for live imaging because it requires fixation and permeabilization and does not provide cell-specific contrast, which has been proven essential for highresolution high-contrast imaging. The primary approach for live visualization of protein dynamics has been to overexpress a fluorescent protein (FP)-tagged target protein [1, 2]. However, protein overexpression can result in undesirable side effects. For example, overexpression of FP-tagged postsynaptic protein PSD-95 has been widely used to study protein dynamics and synaptic organization and mark excitatory synapses [3]. At the same time, PSD-95 overexpression also results in significant off-target effects, including increased number and size of dendritic spines and impaired synaptic plasticity $[4,5]$. Although knock-in of FP tags may label a protein without overexpression, the standard knock-in strategies lead to the global expression of the FP, resulting in poor imaging contrast and lack of cell type specific labeling.

To overcome these challenges, we have developed a conditional knock-in strategy termed endogenous labeling via exon duplication, or ENABLED (Figure 1) [6]. This strategy labels protein from their Ctermini in a manner dependent on the expression of Cre recombinase with nearly ideal properties: highly specific, stoichiometric, compatible with live cell imaging, and with minimal perturbations to protein dynamics and cellular function. This approach is readily amendable to high-contrast cell-specific labeling, cell type-specific labeling, and global labeling, as needed. We have used the ENABLED strategy to specifically and stoichiometrically label endogenous PSD-95 with mVenus or mEGFP in global, sparse, and cell-type specific manners (Figure 2). Extensive characterizations demonstrate that FP-tagged PSD-95 can functionally replace wild-type PSD-95.

Using the PSD-95-ENABLED mouse, we found that synaptic structures, as assayed by PSD-95 ${ }^{\mathrm{mVenus}}$ fluorescence, only loosely correlated with the volume of individual dendritic spines, indicating that the spine volume is not an ideal proxy for synaptic strengths. Furthermore, by specifically labeling PSD-95 in discrete populations of aspiny neurons, we unambiguously marked and examined the dynamics of morphologically-unidentifiable shaft excitatory synapses in living tissue (Figure 3). We found that PSD95 levels correlated with the glutamate sensitivity of individual synapses. By using longitudinal in vivo two-photon microscopy, we found that the excitatory synapses of cortical PV interneurons turns over at a rate much lower than their spiny counterparts in layer $2 / 3$ pyramidal neurons. Overall, the $P S D-95$ ENABLED mouse line may open up novel research avenues for studying the function of excitatory synapses, especially those residing on dendritic shafts. The ENABLED strategy should be applicable to a wide variety of important proteins that are compatible with C-terminal FP-tagging [7]. 
References:

[1] M Chalfie et al., Science 263 (1994), p. 802.

[2] AB Cubitt et al., Trends Biochem Sci 20(11) (1995), p. 448.

[3] E Kim and M Sheng, Nat Rev Neurosci 5(10) (2004), p. 771.

[4] AE El-Husseini et al., Science 290(5495) (2000), p. 1364.

[5] V Stein et al., J Neurosci 23(13) (2002), p. 5503.

[6] DA Fortin et al., J Neurosci 34(50) (2014), p. 16698.

[7] This work was supported by NIH grants to HZ (DP2OD008425, R21NS084315, and R21NS097856) and TM (R01NS081071) and by HHMI.
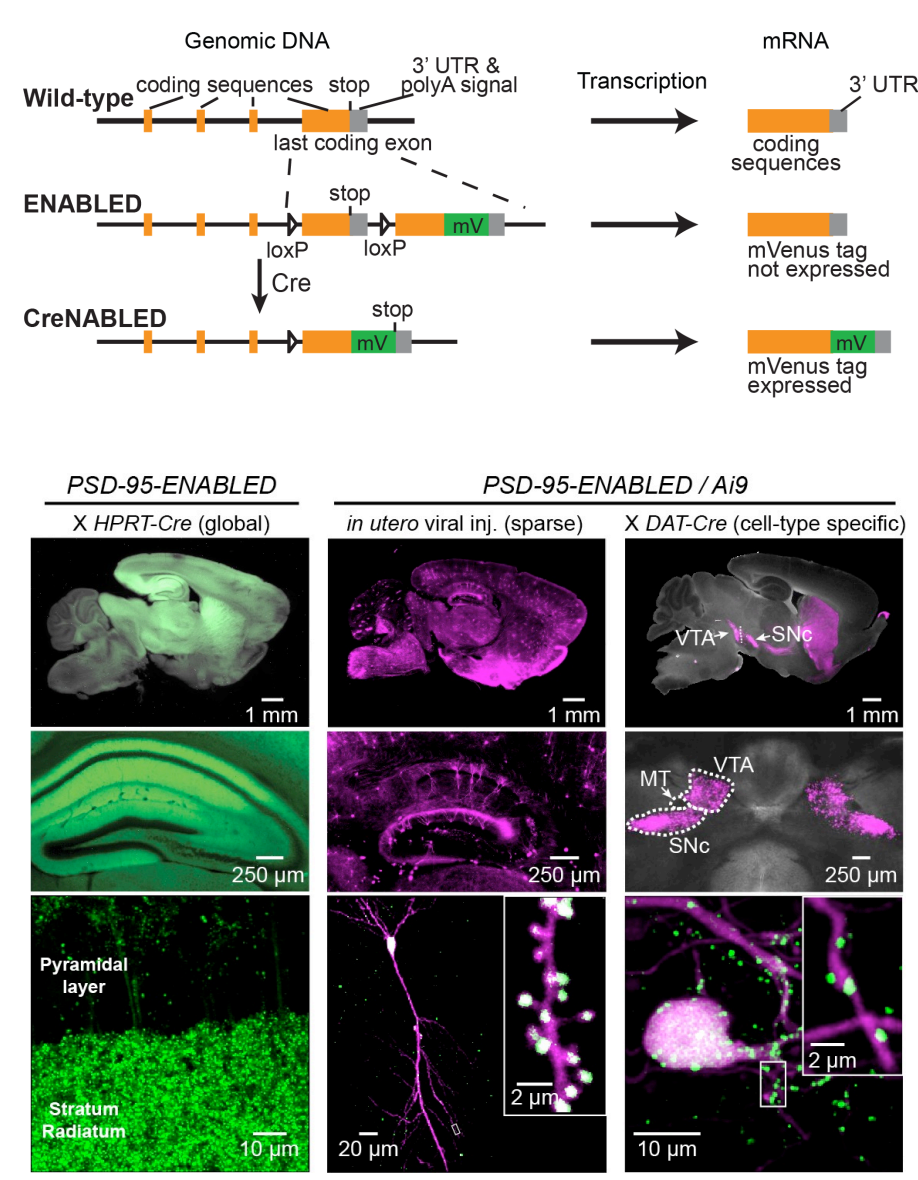

Figure 2. Representative images. PSD-95 is labeled (green) in all neurons, a random sparse subset, and specific neuronal subtypes (example uses the DATCre driver line). Magenta shows tdTomato signal resulting from Cre-dependent recombination of the Ai9 allele.
Figure 1. Design of the ENABLED strategy. The last coding exon of a gene, including the translational stop, 3' UTR and polyadenylation (polyA) signal will be duplicated. mVenus (mV) is fused to the end of the coding region of the duplicated copy. The mVenus tag will not be expressed until Cre/loxP controls mediate the excision of the endogenous exon.
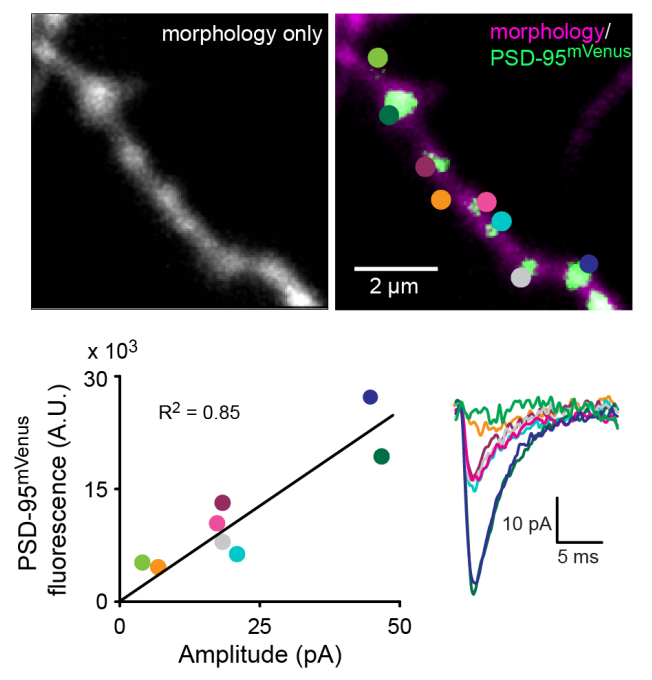

Figure 3. Visualization of excitatory shaft synapses on aspiny neurons. Upper, representative images. Lower, uncaging EPSC amplitudes as a function of PSD$95^{\mathrm{mVenus}}$ fluorescence. Data are colorcoded for the uncaging location in the upper panel. 